\title{
From Evidence to Practice, group education as part of routine outpatient clinic in Polycystic Ovary Syndrome a proof of concept intervention
}

\author{
Hamidreza Mani ${ }^{1,2}$, Ragini Bhake ${ }^{2}$, Michael Pierides ${ }^{1}$, Narendra Reddy $^{2}$, Kishor Patel ${ }^{1}$, Miles J Levy ${ }^{2}$ \\ 1- Department of Diabetes and Endocrinology, Kettering General Hospital NHS Foundation Trust, Kettering, NN16 8UZ \\ 2- Department of Endocrinology, Leicester Royal Infirmary, University Hospitals of Leicester NHS Trust, Leicester, LE1 5WW, UK
}

Background The benefits of patient education in women with polycystic ovary syndrome (PCOS) are known ${ }^{1}$ but a cost effective way to offer the education to these patients need to be assessed.

Aim Incorporating group education for women with PCOS to their routine care process as part of a quality improvement project

\section{Methods}

Setting: Specialist Gynae-Endocrine Clinic in a tertiary teaching hospital

- January-June 2017: Ad hoc open patient invitation (Group A) A researcher (HM) identified all women with PCOS coming to outpatient reception. Patients were given a letter inviting them to a group session. All clinicians and Trainees were encouraged to invite patients to the sessions.

- January - June 2018: Dedicated education clinic (Group B) A clinic code was set up and an official appointment letter sent to patients. All clinicians and Trainees were made aware of the clinic code and availability of the education session through reminder e-mails.

\section{Results}

Figure 1: Comparison of attendance rate in two methods of

- Group A: Ad hoc invitation invitation

135 women with PCOS ( = all women with a diagnosis of PCOS who attended their appointment in the Gynae-Endo clinic) were offered the education session and only $6(4 \%)$ attended.

- Group B: Dedicated education clinic

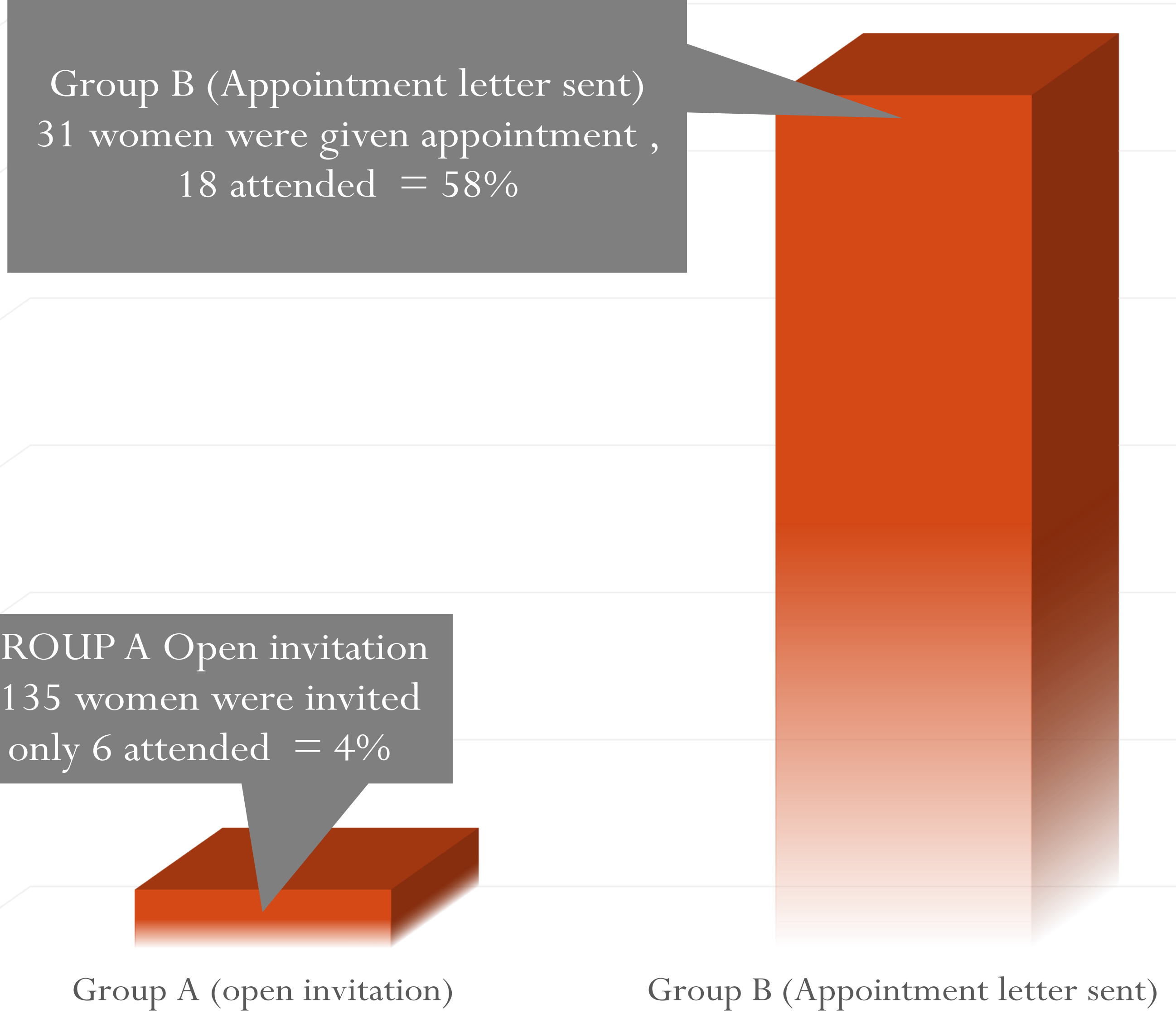

Of potentially 130 women with diagnosis of PCOS who attended their appointment in the Gynae-Endo clinic only 31 were referred to the education session and 18 of them (58\%)

who received an appointment attended the education session.

Figure 2: The dynamic and content of the education session

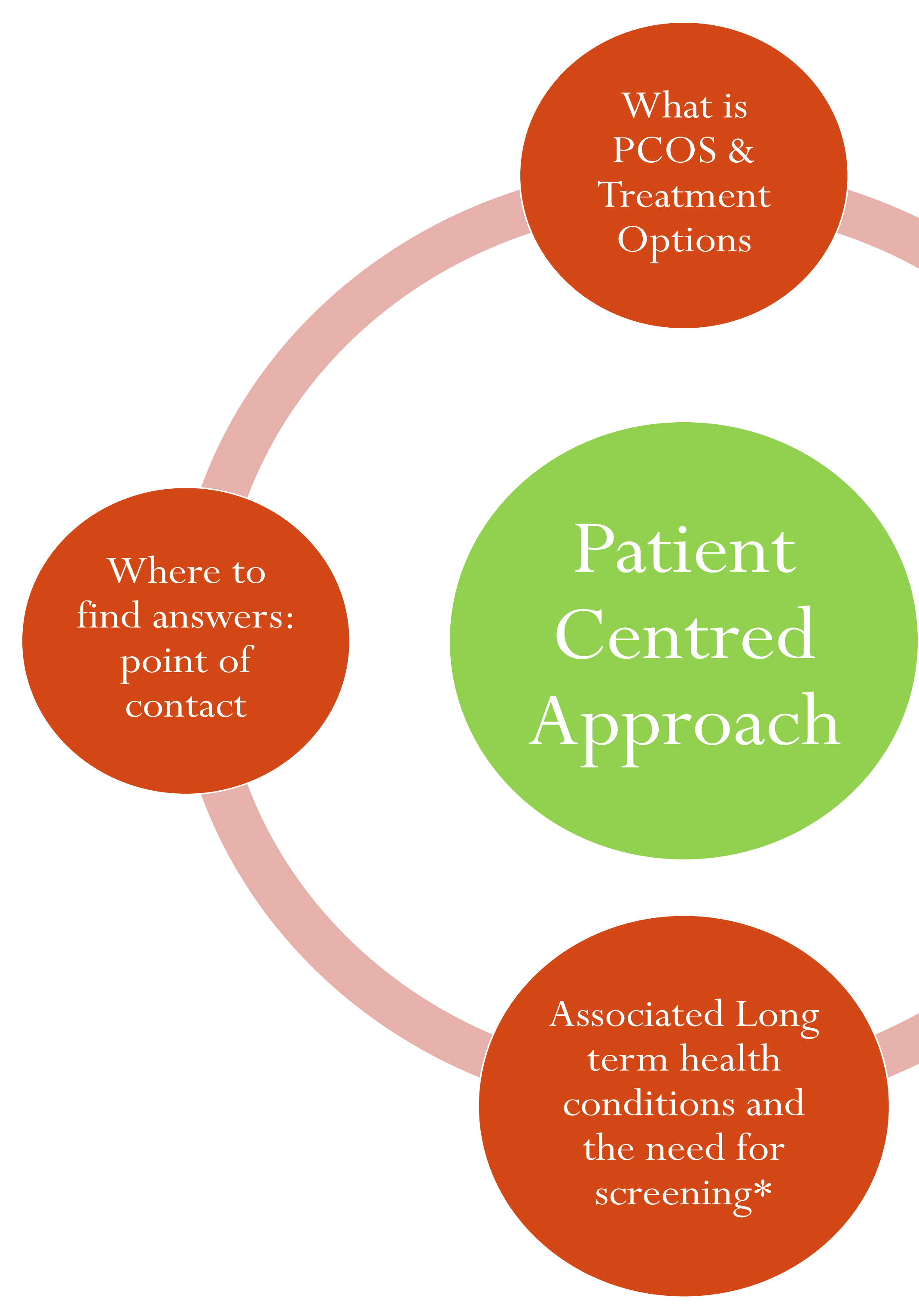

* Such as Type 2 Diabetes, Gestational Diabetes, Depression, Endometrial Cancer, Obstructive Sleep Apnoea, Non Alcoholic Fatty Liver Disease

\section{Patients feedback on education sessions}

All patients but 2 scored the sessions 4 and 5 (good or very good respectively) and expressed a desire for more sessions

After education session, participants felt "better understanding of PCOS” \& “I am not alone"

\section{Discussion and points for debate:}

$>$ Importance of the appointment letter:

In an open discussion with Group B patients who attended the education; they indicated that having an appointment letter in hand helps them to take the required time off work in order to attend the education session.

Understanding PCOS Versus Weight management:

In Group B, less than half of the patients had been referred to education session (31 out of $\sim 130$ ). Perhaps clinicians' understanding of such education sessions need to be addressed: looking at some clinic letters it was obvious that some clinicians only saw the education session as a point for "weight loss discussion" after other usual methods had failed or there was nothing more to offer.While women who attended the education saw the benefits of "understanding PCOS" and "seeing other women with PCOS".

\section{Conclusion}

Patients with PCOS find education sessions helpful and want to come back for more. A dedicated education clinic with an appointment letter with date and time is more effective than an ad hoc open invitation. This proof of concept study might inform methodology for an educational intervention in outpatient setting 